\title{
ADSORPTION EQUILIBRIUM AND KINETIC STUDIES OF PHENOLS AND SUBSTITUTED PHENOLS IN AN AQUEOUS PHASE USING GRANULAR ACTIVATED CARBON
}

\author{
Roy George \\ Department of Chemistry, S.F.S. College, Seminary Hills, Nagpur-440006 \\ E-mail: georgeroysfs@gmail.com
}

\begin{abstract}
:
Adsorption equilibrium and kinetic studies of phenols and substituted phenols at Para position in an aqueous phase was carried out in the present study. The adsorbent used in study was Granular Activated Carbon (GAC). Bituminous type of coal was source of the granular activated carbon (GAC). The mesh size of GAC used in the study was 12X16. GAC used in the study had already examined for surface area pore size and morphology in previous publication. The surface area of GAC was found to be $998 \mathrm{~m}^{2} / \mathrm{g}$ and loosely bounded and of open pores morphology. The adsorbates selected for these studies were phenol and its para substituted derivative namely; phenol, p-Methoxy Phenol and p-Nitro phenol. The order of adsorption for adsorbates was found to be p-Methoxy Phenol > p-Nitrophenol > Phenol. Functional group at para position was found to alter the adsorption behavior.
\end{abstract}

Keywords: Methicillin, staphylococcus, antibiotics and MRSA.

\section{INTRODUCTION:}

Adsorption of pollutants on activated carbon remains better choice for separation, treatment of waste effluent, refrigeration, environmental pollution control etc [1-3]. Organic compounds such as aniline, benzoic acid, phenols and substituted phenols are common organic pollutants, need to be removed from waste water as these compounds are toxic and also posses carcinogenic characteristics [4-8]. One viable technique for scavenging a broad spectrum of organic chemicals, which has emerged as the most economical and efficient method is adsorption on activated carbon. It is now proved through commercial unit that carbon adsorption system consistently producing high level of treatment and have high degree of stability and reliability $[5,9,10]$. Activated carbon is a versatile material. The surface area of GAC and its surface characteristic can be altered subsequently by giving it suitable treatment. The adsorbent used in the present study is granular activated carbon reported in previous publications. [11-19]. Selection of and design of batch, fixed bed and fluidized reactors are dependent on adsorption behavior and equilibrium. Better understanding about the adsorbate -adsorbent interactions will give better rational about reactor design.

Present study is work in continuation to the previous publication [1]; focused on adsorption isotherm and kinetics study of one phenol, pmethoxy phenol and p-nitrophenol.
The present study is dedicated to understand the effect of substitution to phenol at para position in its aqueous phase on granular activated carbon (GAC). Industrially important processes are often concerned with more than one adsorbate species therefore both the mono solute and bi-solute systems have been analyzed.

\section{METHOD AND MATERIAL:}

Double distilled water was used for the study obtained from laboratory set up.

The adsorbates chosen for present work were phenol, p-methoxy phenol and p nitrophenol. All the chemicals were obtained from e-Merk make.

The Granular Activated Carbon (GAC) was obtained from source Bituminous type coal provided by Calgon Co-operation U.S.A.

\subsection{Preparation of stock solutions}

Prior to the preparation of stock solution phenol was extracted from $100 \mathrm{ml}$ petroleum ether by vigorous shaking at $40-50{ }^{\circ} \mathrm{C}[20,21]$. The mixture was allowed to stand for 15-30 minutes. Two distinct layers got separated. The upper colorless layer was decanted. The phenol crystals were separated from the petroleum ether and dried in vacuum oven at $35-40^{\circ} \mathrm{C}$. The purity of phenol was determined by the brominating process as recommended in literature [21].

A $0.1 \mathrm{M}$ stock solution of phenol was prepared by dissolving $4.8610 \mathrm{~g}$ of purified phenol in 
freshly boiled and cooled $500 \mathrm{ml}$ distilled water.

A $0.1 \mathrm{M}$ solution of p-methoxy phenol was prepared by dissolving $0.2230 \mathrm{~g}$ of $\mathrm{p}$ methoxyphenol in $500 \mathrm{ml}$ double distilled water.

A $0.1 \mathrm{M}$ solution of $\mathrm{p}$-nitrophenol was prepared by dissolving $6.9555 \mathrm{~g}$ of $\mathrm{p}$-nitrophenol in 500 $\mathrm{ml}$ double distilled water.

The concentrations of solutes present in stock solutions were standardized by using ultraviolet adsorption (UV) spectroscopy by measuring the optical density of the component at their respective wavelength of maximum absorbance.

\subsection{Characterization:}

The surface area, pore volume, pore size and morphology of adsorbent GAC was estimated and reported in previous publication.

In the present study Grannular activated carbon was investigated by using Fourier Transform Infra Red (FTIR) analysis. The FTIR analysis was carried out on a Nicolet Magna-IR 550 spectrophotometer series II.

The concentration of adsorbate in the solution was estimated by UV adsorption by measuring the optical density of the component at their respective wavelength of maximum absorbance using SPEKTROMOM 210 (Hungarian Optical Works, MOM, Budapest).

\subsection{Experimental methods for evaluation of adsorption equilibrium:}

The experimental set up for evaluation of adsorption equilibrium is shown previous publication [11]. However, the method and arrangement in short is provided here. Electrical power supply of $1 \mathrm{KVA}$ and $5 \mathrm{kVA}$ was obtained from voltage stabilizer ( M/S Toshniwal Ptt. Ltd, Mumbai, India). A 45 liter plastic water container was used as water bath equipped with Remi Stirrer ( Type L-157, Remi Udyog, Mumbai2, India). The bath was heated to $110^{\circ} \mathrm{C}$.

For the equilibrium runs, GAC/Adsorbate solution ratio by volume was kept constant at $0.50 \mathrm{~g} / 500 \mathrm{ml}$ in all equilibrium experiments. In particular 1 Liter round bottom flask containing $500 \mathrm{ml}$ of distilled water was immersed in the thermostat bath with constant stirring for $30 \mathrm{~min}$. The impeller made up of glass with dimensions of $6 \mathrm{~mm}$ thickness and $4 \mathrm{~cm}$ length was used. As the temperature of $30{ }^{\circ} \mathrm{C}$ was reached, calculated quantity of the stock solution measured with the help of measuring pipette, was introduced into the distilled water, and allowed to mix thoroughly to achieve respective concentrations. Aliquot of resulting solution was pipette out in order to determine initial concentration. $0.5 \mathrm{~g}$ of granular activated carbon sample was introduced into the solution with stirring at $1000 \mathrm{rpm}$. The stirring was continued till the concentration of the aqueous phase showed no detectable change as measured by UV absorbance. The equilibrium concentration was calculated from data available about molar extinction coefficient of respective adsorbates. Experiments were continued for $5 \mathrm{hrs}$ in order to ensure complete equilibrium..

For a bi-solute system a similar concentration range of stock solution of the constituent solute in equal concentrations were added. Rest of the experimental set up and method was same as above stated.

\subsection{Experimental method for evaluation of adsorption kinetics:}

The experimental set up for adsorption kinetic study is described in detailed in previous publication. For adsorption kinetic studies, a batch reactor of 8 Liters capacity made up of plexi glass equipped with baffle was used. The GAC adsorbate system was stirred by the bladed impeller of length $7 \mathrm{~cm}$ and breadth 1.5 $\mathrm{cm}$. Experimental solutions for kinetics runs was $4 \mathrm{~L}$ in volume and was prepared by adding appropriate amounts of stock solution to fresh distilled water. $2 \mathrm{gm}$ of GAC was introduced into the solution at a given instant of time. At intermittent interval of time, $5 \mathrm{ml}$ of experimental solution was withdrawn with the help of a syringe and the concentration of adsorbate in the aqueous phase was estimated by UV analysis.

\subsection{Bi-solute adsorption experiment:}

While selection of bi-solute adsorption experiments solutes were selected so that respective adsorption was sufficiently apart so as to permit their simultaneous quantitative estimation. An adsorbate from acid family studied in previous publication was selected for bi-solute adsorption experiment along with phenol and substituted phenol.

\subsection{Irreversibility of adsorption}

The irreversibility of adsorption was confirmed by process in which at the end of kinetic experiment adsorbate and adsorbents are separated using filtration. Residue was washed several times. Washed and dried adsorbent 
was introduced the same quantity of distilled water with constant stirring for $5 \mathrm{~h}$. Aliquots of samples were withdrawn and monitored on UV spectrometer intermittently.

\section{RESULT \& DISCUSSION}

The nature of adsorption isotherm gives qualitative information about adsorption process and the extent of surface coverage by the adsorbate.

In the present work single and multicomponent adsorption equilibrium and kinetic studies have been carried out using phenol, $p$ methoxy phenol and p-nitro phenol. The solubility of these compounds and their stability in water were also taken into account.

3.1. Characterization of Granular Activated Carbon

The Granular Activated carbon was studied in detail for its physical characteristics in previous publication. However, for instance its characteristics are mentioned.

The Granular activated carbon used in the present study was obtained from bituminous coal and was analysed for BET-Surface are, morphology proximate and ultimate estimation in previous publication. The respective values are included here for reference purpose. BETsurface area of GAC was $998 \mathrm{~m}^{2} / \mathrm{g}$. The particle density of GAC was $0.7950 \mathrm{~g} / \mathrm{cm}^{3}$. Pore volume of $0.825 \mathrm{~cm}^{3} / \mathrm{g}$ and pore diameter less than 20 AU was observed for GAC. SEM analysis exploits morphology of GAC which is loosely packed with lots of cavities, cracks, irregular protrusion and widely dispersed pores. The GAC used in the study comprises of $1.9 \%$ moisture, $6.1 \%$ ash, $2.7 \%$ volatile matter, $89.3 \%$ of fixed carbon, $6.71 \%$ of mineral matter according to proximate analysis.GAC used in the study was found to contain $94.04 \%$ of $\mathrm{C}$ and $0.29 \%$ of hydrogen by ultimate analysis. The GAC of mesh size $12 \mathrm{X} 16$ was used.

\subsubsection{FTIR analysis}

The FTIR spectra of GAC samples in a $\mathrm{KBr}$ matrix was recorded between $4000-400 \mathrm{~cm}^{-1}$ at room temperature is shown in Fig.1. The presence of various surface groups was observed. The predominant presence of free phenolic $-\mathrm{OH}$ stretching vibration around 3500 $\mathrm{cm}^{-1}$ was observed. The band at 2900-3000 $\mathrm{cm}^{-1}$ is due to asymmetric and symmetric $\mathrm{C}-\mathrm{H}$ stretching vibrations. The spectrum around $1000 \mathrm{~cm}^{-1}$ and below is marked by the noisy spectrum in this region. The band at $1629 \mathrm{~cm}^{-1}$ represents $\mathrm{H}-\mathrm{OH}$ bonding vibrations.

\subsection{Adsorption equilibrium study}

According to Langmuir adsorption isotherm maximum adsorption corresponds to a saturated monolayer of adsorbate molecule on adsorbent surface. The energy of adsorption is constant and that there is no transmigration of adsorbate in the plane of the surface.

The equation can be expressed as

$Q e=\frac{Q o b C e}{1+b C e} \ldots \ldots(1)$
$\frac{1}{Q e}=\frac{1}{Q 0 b C e}+\frac{1}{Q o} \ldots \ldots(2)$

Where,

$\mathrm{Q}_{\mathrm{e}}=$ Amount of adsorbed solute per unit weight of adsorbent at equilibrium $(\mathrm{mol} / \mathrm{g})$

$\mathrm{C}_{\mathrm{e}}=$ Equilibrium concentration of adsorbate in solution (mol/L)

$\mathrm{Q}_{\mathrm{o}}=$ Monolayer capacity of adsorbent $(\mathrm{Mol} / \mathrm{g})$

$\mathrm{b}=$ Langmuir constants

If plot of $1 / \mathrm{Qe} \mathrm{Vs} 1 / \mathrm{Ce}$ is linear implies Langmuir adsorption isotherm is operative.

Another important adsorption isotherm is

Freundlich adsorption Isotherm. The equation for Freundlich adsorption isotherm is $\log \mathrm{Qe}=\log \mathrm{k}+\mathrm{n} \log \mathrm{Ce}----------(3)$

If plot of $\log$ Qe Vs $\log$ Ce is linear implies Freundlich adsorption isotherm does hold. .

Multi-solute adsorption system can be explained extending known isotherm such as Langmuir- Freundlich and Ideal Adsorbed Solution Theory (IAS).

\subsubsection{Single solute adsorption}

The adsorption equilibrium isotherm plots for phenol, p-methoxy phenol and p-nitrophenol on GAC of mesh size $12 \mathrm{X} 16$ are shown in Fig. 2 This figure depict plot of Qe (amount of adsorbate on the adsorbent, $\mathrm{mol} / \mathrm{g}$ ) Vs the equilibrium concentration of adsorbate in solution $\mathrm{Ce}(\mathrm{mol} / \mathrm{L})$ fairly indicate typical type 1 Favourable isotherm as generally referred to in literature [18]. The isotherm exhibits a sharp increase in adsorbent phase concentration in the low adsorbate equilibrium concentration range and plateau at high bulk solution concentration. All adsorption isotherm obtained in the present study fall under this category. Reproducibility of the experimental values has been checked. They were checked by running the experiment for prolonged periods of timing several days where no change in the equilibrium concentration values could be observed.

At equilibrium the concentration of the adsorbate on the adsorbent and the adsorbate in solution is a measure of the position of equilibrium in the adsorption process. A 
relation which relates these two quantities is called an adsorption isotherm under isothermal conditions. Adsorption isotherms can be used to describe and interpreted the influences with various parameter exhibit upon the adsorption process. Both the Freundlich and the Langmuir adsorption equilibrium model were used to describe the adsorption characteristics of aromatic organic pollutants. In the present investigation, an evaluation was made to determine if the experimental data for different adsorbate could be fitted to either the Langmuir or Freundlich equations. The adsorption equilibrium data for single solute-adsorbent system were hence treated by both Langmuir and Freundlich isotherm equation. Plots for both Langmuir and Freundlich isotherms for systems had obtained and shown in Fig 3 and 4. The result from this analysis indicated that the experimental data did not adhere to either of these two models completely. However, the data could be fitted to either model prided one was selective in the equilibrium concentration range to be used in the analysis. Table 1 gives the complete isotherm equation, Langmuir and Freundlich constant as well as the equilibrium adsorbate concentration Ce range over which the given system adhere to the linearity of the relation for all adsorbent -adsorbate systems studies. The isotherm equations were obtained by the method of linear regression of the experimental adsorption equilibrium data. The values of the regression coefficient $(\mathrm{r})$, for all the adsorbent-adsorbate systems was found to be in the range of 0.97 to 0.99 , indicating a good mathematical fit since for a given system both isotherms were mathematically fitted as shown in Table 1. The equation which was applicable over a large adsorbate concentration range was taken as the one representing the isotherm for the adsorbent -adsorbate system and used in further calculations.

\subsubsection{Multi-solute adsorption equilibrium:}

The adsorption equilibrium obtained for various bi-solute-adsorbent system is shown in Fig. 5. The removal rate for all the components was much higher for bi-solute systems. Several bi-solute adsorption equilibrium and kinetic studies were carried out to delineate the influence of competing adsorbates on the adsorption process. It is assumed that various solutes diffuse independently of one another and the competitive effects are taken into account only through the adsorption isotherm. Thus to evaluate the competitive effect of various adsorbates, the multisolute adsorption equilibrium data was analysed by Langmuir model. For the evaluation of Qo and $b$ values, the adsorption equilibrium data for all components in bisolute system were treated independently to verify the validity of the Langmuir equation. The change in the adsorptive capacity of an adsorbate on a particular grade of the adsorbent when present in multisolute system vs the adsorptive capacity of the solute from its single solute system was compared to observe the competitive effects. The obtained values of Qo and constant $b$, for all the bisolute systems are reported in Table 2.

If we compare the monolayer capacity of each adsorbate on a particular adsorbent then it becomes apparent that the monolayer adsorption capacity for each adsorbate in a solute system gets reduced relative to its value in the single solute system but the cumulative adsorption capacity for multisolute system were higher than those for single solute system. In the mixture, the competitive effects are more pronounced as the number of solute increase and the total adsorption capacity is thus enhanced due to increased cumulative adsorbate concentration.

The overall removal rate of adsorbate is reported to increase with increasing initial adsorbate concentration. Increasing in the initial concentration of phenols, decreases break through time. Presence of other solute is advantageous because a greater utilization of adsorbent can be obtained.

\subsection{Kinetics of adsorption}

The comparative adsorption kinetics plot for bi-solute adsorbates under this study is shown in Fig.6. For bi-solute adsorbates first component was salicylic acid and second component was respective phenols. The study indicates that adsorbates were removed very rapidly from the aqueous phase within the first hour of contact. In the case of competitive adsorption involving two solutes, it is assumed that the various solutes diffuse independently of one another and the comparative effects are taken into account only through the adsorption isotherm Rate constant found in this manner are purely phenomenological depending upon the rate of adsorption and diffusion. They found useful in comparision between various adsorbates. The individual process like adsorption, desorption and molecular diffusion are all coupled together and represented by overall rate constant. 
The adsorption kinetic data was fitted in the equation

$\ln (\mathrm{C}-\mathrm{Ce}) /(\mathrm{C}+\mathrm{D})=-\mathrm{kt}$; Where, $\mathrm{D}=\mathrm{Co} / \mathrm{K} \mathrm{Ce} \ldots . .(4)$

Adsorption kinetics studies for single soluteadsorbent system shows that the adsorbate removal rate for the carboxylic acid decreases as their solubility in aqueous phase increases. Detail discussion about effect of solubility on adsorption is discussed in further section. During the competitive adsorption, the more hydrophobic adsorbates was removed at a faster rate as compared to the less hydrophobic one. In the mixture, the competitive effects are more pronounced the number of solute increases and the total adsorption capacity was thus enhanced due to increased cumulative adsorbate concentration. According to the literature the increasing initial concentration of a solute decreases the time at which the breakthrough appears. The competitive adsorption from mixture of organic may be advantageous or detrimental depending on intended application of the adsorption unit. If the purpose is complete removal of all organics, then presence of other solute is advantageous because of a greater utilization of the adsorbent can be obtained whereas; if the application is to selective removal of a certain organic compound, then the efficiency of the GAC will decrease with increase in concentration of the adsorbate solute.

\subsection{Effect of functional group at para position}

A type of functional group present on adsorbate is an important as its size and configuration. The addition of substituent group on adsorbate can manifest itself in several ways. First, the substituent group may alter the solubility of the adsorbate in aqueous solution. Since the solubility is a measure of adsorbate solvent forces of attraction any change in solubility of molecule will influence the adsorbate-adsorbent forces of attraction. Secondly the adsorbate may interact specially with a compatible functional group present on the carbon surface. Thirdly, the addition of certain functional group to the benzoic acid group will alter the electron density of the benzene ring. This influence is significant only if the $\Pi$ electrons of the adsorbate molecule are involved in the mechanism of adsorption. Finally the substituent group may alter the acidic dissociation constant of $\mathrm{OH}$ group of phenolic compound decides whether or not the adsorbate will be in associated or dissociated form.

\subsection{Effect of molecular weight on adsorption capacity}

The adsorption capacity for phenol, pMethoxy-phenol and p-Nitrophenol on GAC with respect to molecular weight is shown in Fig 7. In general tendency of increase in adsorption capacity with increase in molecular weight of adsorbate, but in this study no such clear trend could be observed. Apparently pMethoxy Phenol and p-Nitro phenol were adsorbed at higher level.

\subsection{Effect of solubility of adsorbate on adsorption:}

The solubility of adsorbates at experimental conditions was observed and it was found to be $0.849 \mathrm{~mol} / \mathrm{L}$ for phenol, that of $\mathrm{p}$-Methoxy Phenol was found to be $0.12 \mathrm{~mol} / \mathrm{L}$, that of $\mathrm{p}$ Nitro Phenol was $0.390 \mathrm{~mol} / \mathrm{L}$.

The solubility of the adsorbate is also known to affect the overall adsorprtion capacity to the large extent. The pure activated carbon surface is considered to be non-polar and is generally regarded to favor the adsorption of non-polar solutes. However, as indicated by the FTIR spectra some phenolic $\mathrm{OH}$ is present on the surface which render the surface slightly polar. Solubility trend of phenols and substituted phenol have order of p-Methoxy Phenol < pNitro Phenol < Phenol. That of adsorption isotherm follows order of p-Methoxy Phenol $\approx$ p-Nitro Phenol > Phenol. The observation implies that more hydrophobic solutes are adsorbed to a greater extent from its aqueous solution. Which implies that the physicchemical properties associated with adsorbate is playing important role in the overall adsorption process.

The possibility of hydrogen bonding between the solute and water molecule has a major effect on the solute adsorption by activated carbon and hence the molecular weight and solubility should not necessarily be considered as a controlling factor. The low adsorptive capacity phenol may be attributed to the ability of $\mathrm{OH}$ group to form a hydrogen bond with water, decreasing the solvophobic nature of the solute, whereas any substituent group in phenol molecule might be preventing the formation of hydrogen bond between the solute and solvent, either by forming internal hydrogen bond between $-\mathrm{OH}$ and other substituent group or by offering steric hindrance, thereby increasing the solvo phobic 
nature as well as sorption capacity of the solute.

The groups introduced in phenol molecules are methoxy and nitro group at para position. PMethoxy phenol and p-Nitro phenol effectively desorbs phenol from the adsorbent surface. The introduction of the substituent groups improved the adsorption. i.e. introduction of such groups hinders the possibilities of the hydrogen bonding with solvent, water; thereby decrease the hydrophilic character.

The effect of introduction of substituent groups in phenols at para position also increases the adsorption capacity. The substitution of electron withdrawing groups like the nitro in phenol lowers the electron density in the pisystem of the ring which act as an electron acceptor, hence uptake of PNP is much more than phenol. $\mathrm{OH}$ group points towards the surface of water and the aromatic ring points vertically away from the solution.

\subsection{Competitive adsorption equilibrium}

The predicted isotherm by Langmuir equal competition and unequal competition models as well as the extended freundlich isotherm model. The predicted isotherm from Langmuir model deviated to larger extent. In contrast to this, the extended Freundlich isotherm model fits the experimental fairly well at lower adsorbate equilibrium concentration ranges and tends to show a positive deviation at adsorbate equilibrium concentration values.

\subsection{Irreversibility experiments:}

From the irreversibility experiments it can be observed that there was no detectable change in transmission values showing that the adsorbate is firmly adsorbed on the adsorbent and the process is irreversible. However the adsorbent was stirred in spectroscopic grade methanol the adsorbate was released into the solution showing thereby that the adsorbate could be desorbed from adsorbent by methanol, which is used as for regeneration of GAC.

\subsection{Stability of adsorbent:}

The GAC used in experiments were stable during the course of experimentation and did not show any change in size during runs.

\section{CONCLUSION:}

This study includes the Adsorption Equilibrium and Kinetic Studies of Phenol, pMethoxy phenol and p-Nitro-Phenol in an aqueous phase using Granular Activated
Carbon. GAC used in the present study was same to that reported in previous publication. Characterization tool Fourier Transform Infra Red (FTIR) spectroscopy was used to examine the GAC. Predominant presence of free $-\mathrm{OH}$ stretching and Adsorbed $\mathrm{CO}_{2}$ was observed. Adsorption is very rapid in first hour of contact, where, $60-80 \%$ of adsorbate was removed followed by slow approach to equilibrium. The adsorption isotherm obtained for various adsorbate -adsorbent system indicates a sharp increase in adsorbent phase concentration in the low adsorbate equilibrium concentration range and plateau at high bulk solution concentration. The experimental isotherm did not adhere to either Freundlich or Langmuir models completely. However, the data could be fitted well to either model if one is selective in the equilibrium concentration range to be used in analysis. Overall adsorption order for phenol and its para substituted derivative in its aqueous phase over Granular Activated Carbon was found to be p-methoxy phenol > p-nitrophenol $>$ Phenol. The adsorption capacity and removal rate of individual components detected as relative to its value in single solute systems but the cumulative adsorption capacity and adsorbate removal rate increased for bisolute system

\section{Acknowledgement}

Author acknowledges Dr G.S. Natarajan and Dr. Mahesh Yenkie for guidance. Author also acknowledges to Laxmi Nrayan Institute of Technology and RTM Nagpur University for providing space and infra structure to carry out experiments. Author acknowledges Calgon Co-operation U.S.A. for providing Granular Activated Carbon.

\section{REFERANCE :}

A. Esposito, J. Raffaelli, L. Fassino, H. S. Odetti and E. J. Bottani, J. the Argentine Chem.Soc., 91, 73 (2003).

S. Sohn, D. Kim, Chemosphere, 58, 115 (2005).

R. K. Rajoriya, B. Prasad, I. M. Mishra, K. L. Wasewar, Chem. Biochem. Eng. Q. 21, 219 (2007). 
L. T. Markovska, V. D. Meshko, M. S. Marinkovski, J. Serbian Chem. Soc., 71, 957 (2006).

A. Meghea, H. H. Rehner, I. Peleanu, R. Mihalache, J. Radioanalytical Nuclear Chem. 229, 105 (1998).

R. Panahi, E. Vasheghani-Farahani, S. A. Shojosadati, Iranian J. Chem. Chem. Eng., 5,49 (2008).

C. Baggiani, G. Giraudi, C. Giovannoli, C. Tozzi, L. Anfossi, Analytical Chimica Acta, 504, 43 (2004).

S. Basu, P. F. Henshaw, N.Biswas and H. K. Kwan, Canadian J. Chem. Eng., 80, 1 (2002).

Journal of the Institution of public health Engineering India, vol. 1977 No:4

V. K. Verma and A.K., Mishra, Global NEST J., 12 , 190 (2010).

R.George, Asian Journal of Chemistry, 31, 1667 (2019).
K. Sartova, E. Omurzak, G. Kambarova, I. Dzhumaev and Z.Abdullaeva, 91, 90 (2019)

H. Wang, B. Wang, J Li, T. Zhu, Separation and Purification Technology, 209, 535 (2019),

F. Suo, X. Liu, C. Li, M. Yuan, M. Ji, International Journal of Biological Macromolecules, 121806 (2019),

B. Li, C. Ma, Energy Procedia, 153, 471 (2018)

H-J Kim, Y-K Han, Current Applied Physics, 16, 1437 (2016).

N. Dejang, O. Somprasit, S. Chindaruksa, Energy Procedia, 79, 727 (2015)

M. Açıkyıldız, A. t Gürses, S. Karaca, Microporous and Mesoporous Materials, 198, 45 (2014)

C.L., Chuang, P.C., Chiang, E.E., Chang, Chemosphere, 53, 17 (2003).

P.H.Mars, Pharm werkblad, 88, 319 (1953).

D.D. Perrin, W.L.F. Armargo, D.R.Perrin, Pergamon Press Ltd, Oxford, London (1966).

Table 1 : Langmuir and Freundlich isotherm data for single solute system.

\begin{tabular}{|c|c|c|c|c|c|c|c|}
\hline \multirow[b]{2}{*}{ System (mesh size) } & \multicolumn{4}{|c|}{ Langmuir isotherm } & \multicolumn{3}{|c|}{ Freundlich isotherm } \\
\hline & $\begin{array}{c}\text { Equation } \\
(\mathbf{Q e}=)\end{array}$ & $\mathbf{r}$ & $Q$ (mmol/g) & $\begin{array}{c}\mathrm{Ce} \\
(\mathrm{mmol} / \mathrm{L})\end{array}$ & $\begin{array}{l}\text { Equatio } \\
\text { n }(Q \mathbf{Q e}=)\end{array}$ & $\mathbf{r}$ & $\mathrm{Ce}(\mathrm{mmol} / \mathrm{L})$ \\
\hline Phenol (12X16) & $\frac{7.8861 C e}{1+3233 C e}$ & 0.99 & 2.44 & $\begin{array}{c}0.0106- \\
0.4\end{array}$ & $\begin{array}{l}8154 \mathrm{Ce}^{0 .} \\
7996\end{array}$ & 0.98 & $0.0034-0.42$ \\
\hline $\begin{array}{l}\text { p-Nitrophenol } \\
(12 \times 16)\end{array}$ & $\frac{37.4405 C e}{1+12260 C e}$ & 0.98 & 30.5371 & $0.05-5.42$ & $\begin{array}{c}182.3 \\
\mathrm{Ce}^{0.2796}\end{array}$ & 0.99 & $0.018-0.47$ \\
\hline $\begin{array}{l}\text { p-Methoxy phenol } \\
\text { Acid(12X16) }\end{array}$ & $\frac{20.974 C e}{1+8943 C e}$ & 0.99 & 23.45 & $\begin{array}{l}0.073- \\
0.585\end{array}$ & $\begin{array}{c}284.3 \\
\mathrm{Ce}^{0.3517}\end{array}$ & 0.97 & $0.07-0.585$ \\
\hline
\end{tabular}

Table 2 : Langmuir constants for bisolute system.

\begin{tabular}{|c|c|c|c|c|c|c|}
\hline $\begin{array}{l}\text { System (mesh } \\
\text { size) }\end{array}$ & $\begin{array}{c}\text { Qo1X } \\
10^{-4}(\mathrm{~mol} / \\
\mathrm{g})\end{array}$ & b1 & $\begin{array}{c}\text { Qo1X } \\
\text { 10-4(mol/ } \\
\mathrm{g})\end{array}$ & b2 & $\begin{array}{c}\text { (Qo1+Qo2) } \\
\times 10^{-4} \\
(\mathrm{Mol} / \mathrm{g})\end{array}$ & (b1+b2) \\
\hline $\begin{array}{l}\text { Salicylic acid- } \\
\text { Phenol (12X16) }\end{array}$ & 23.98 & 3357 & 6.77 & 1268 & 30.74 & 4625 \\
\hline $\begin{array}{ll}\text { Salicylic } & \text { acid-p- } \\
\text { Methoxy } & \text { phenol } \\
(12 X 16) & \end{array}$ & 34.54 & 239 & 13.22 & 2440 & 47.96 & 2679 \\
\hline $\begin{array}{l}\text { Salicylic acid-p- } \\
\text { Nitrophenol(12X1 } \\
\text { 6) }\end{array}$ & 15.52 & 19514 & 14.59 & 21274 & 30.13 & 40788 \\
\hline
\end{tabular}



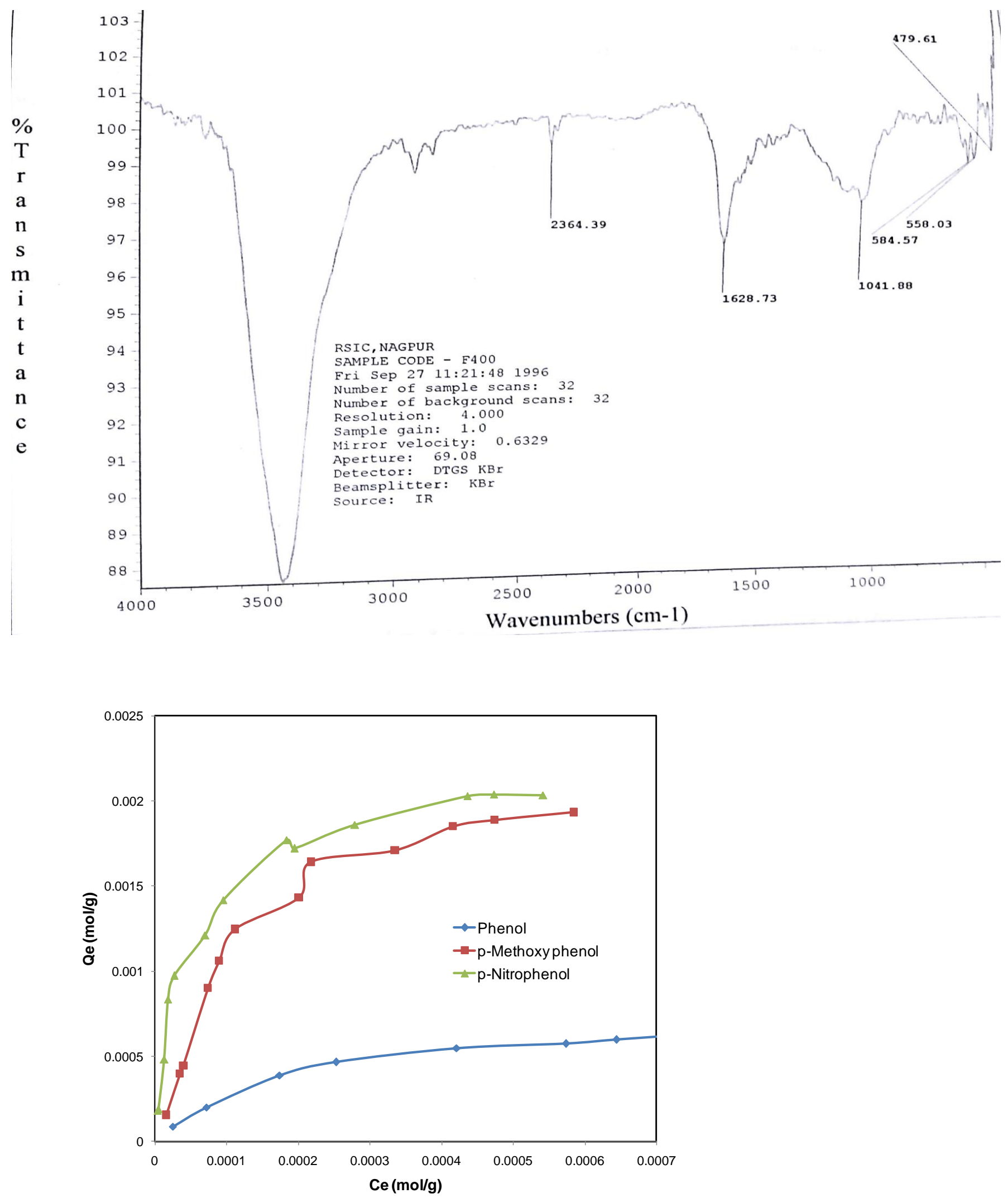


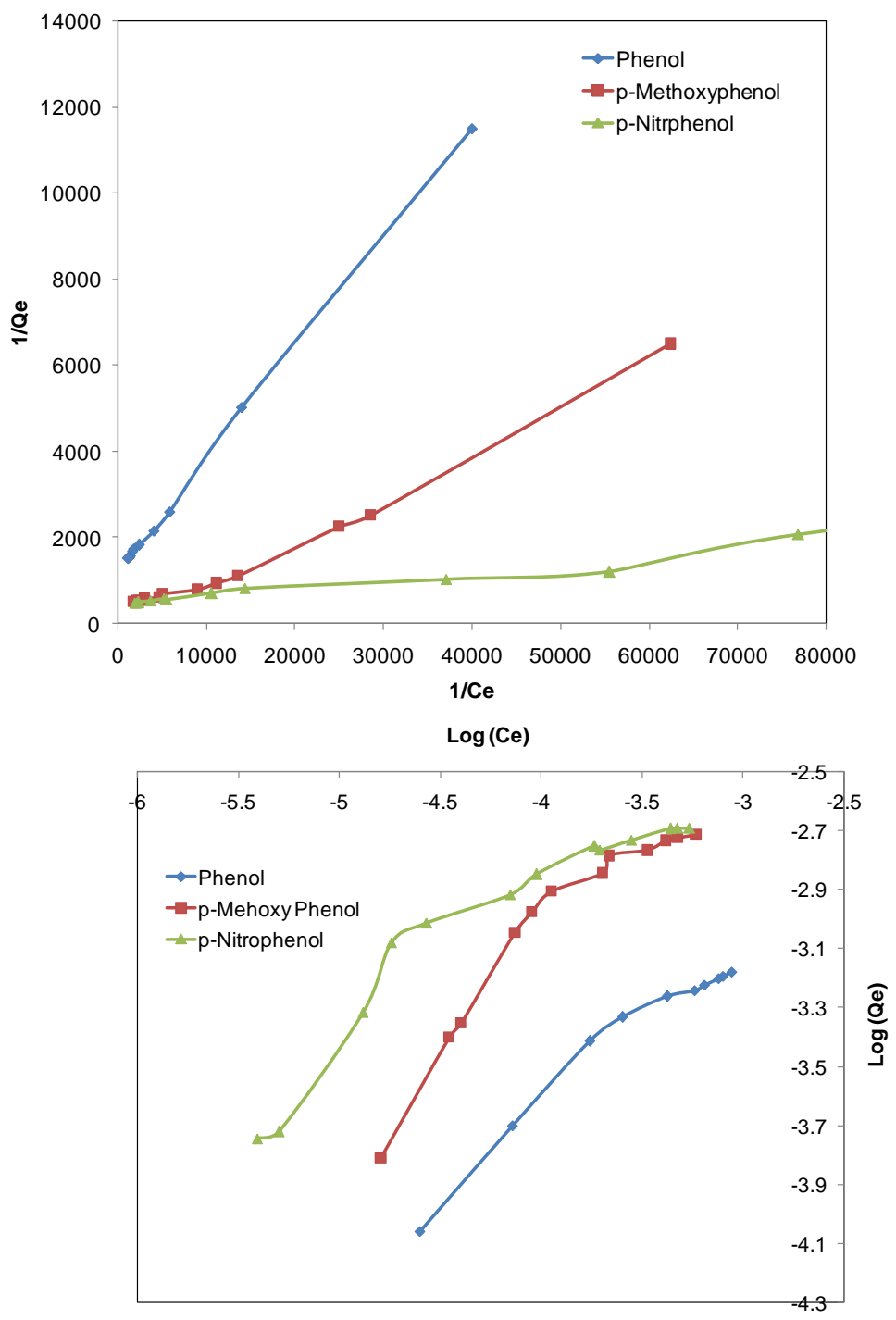

Fig : 4

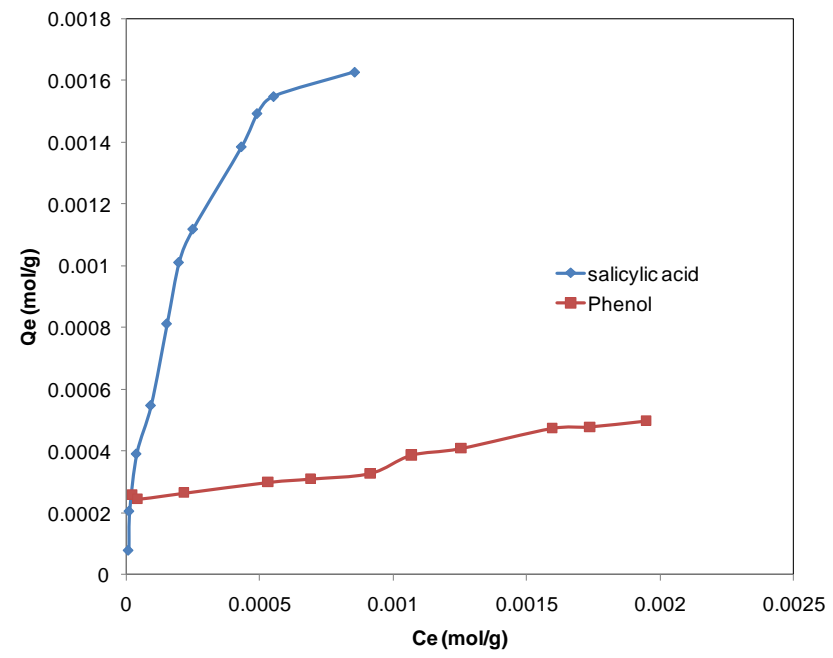

Fig 5 (a) 


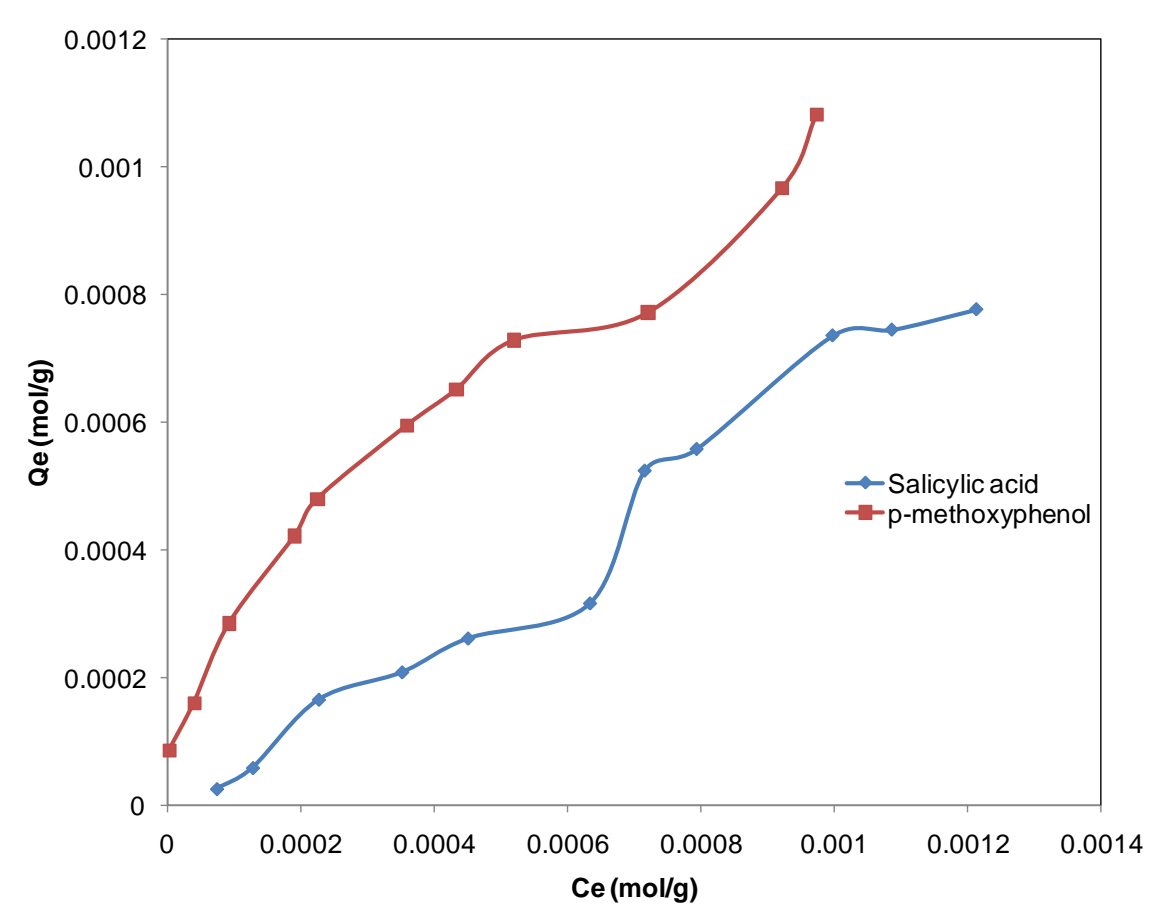

Fig : 5 (b)

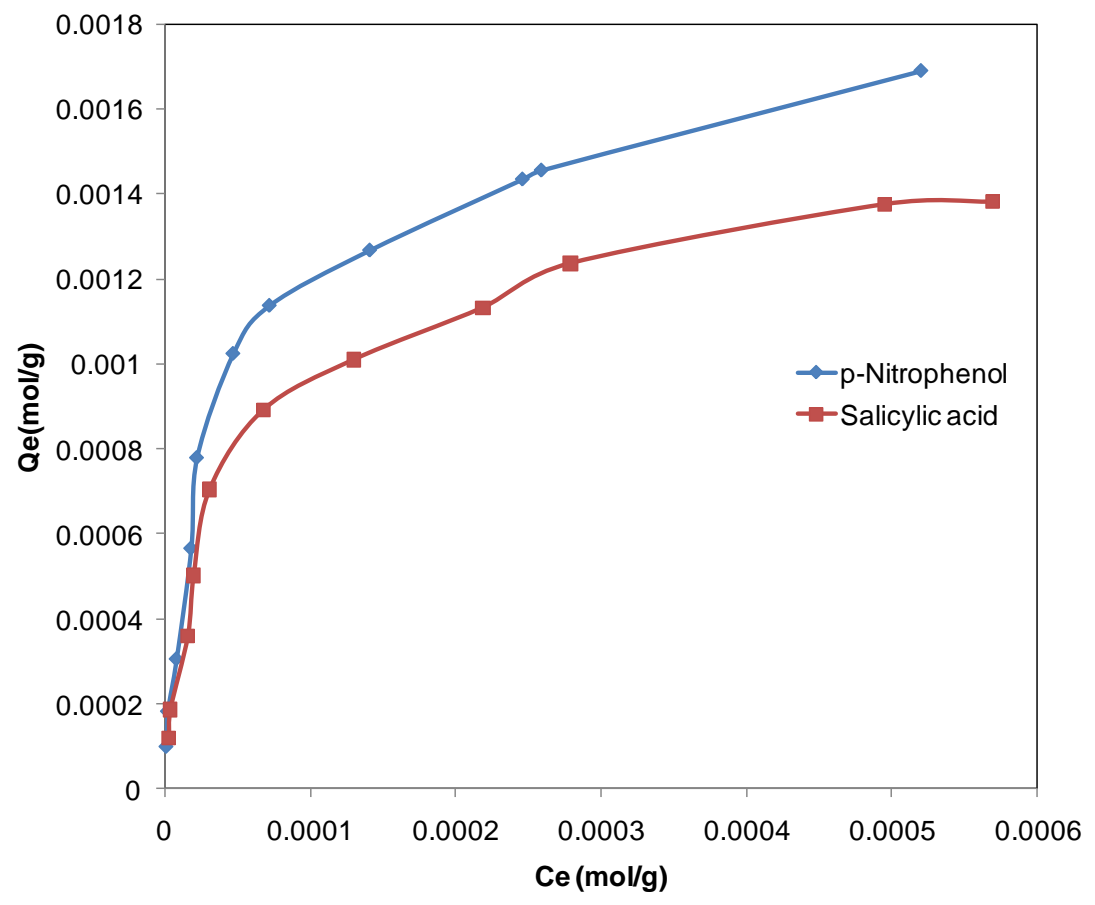

Fig : 5 (c) 


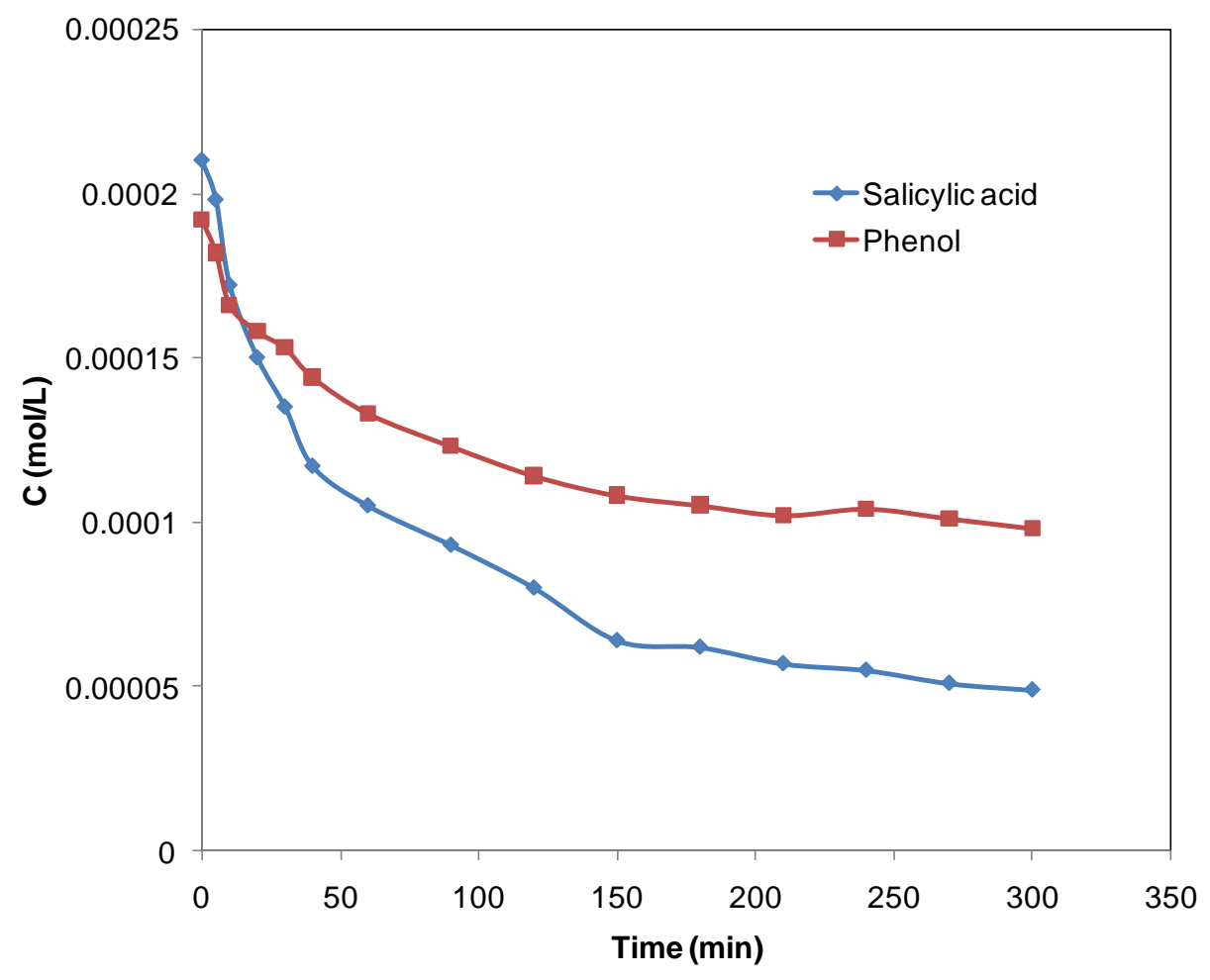

Fig. 6 (a)

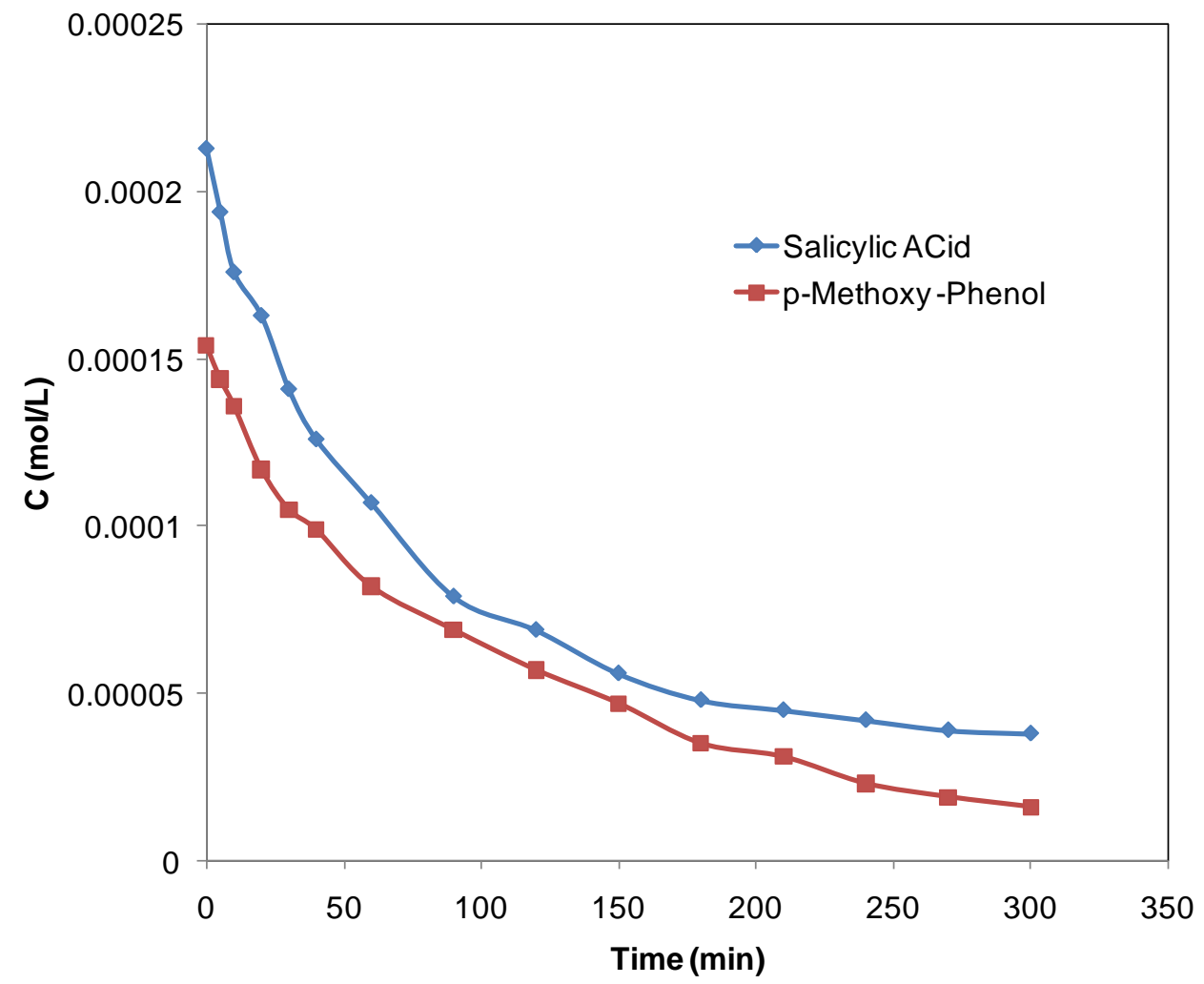

Fig. 6(b) 


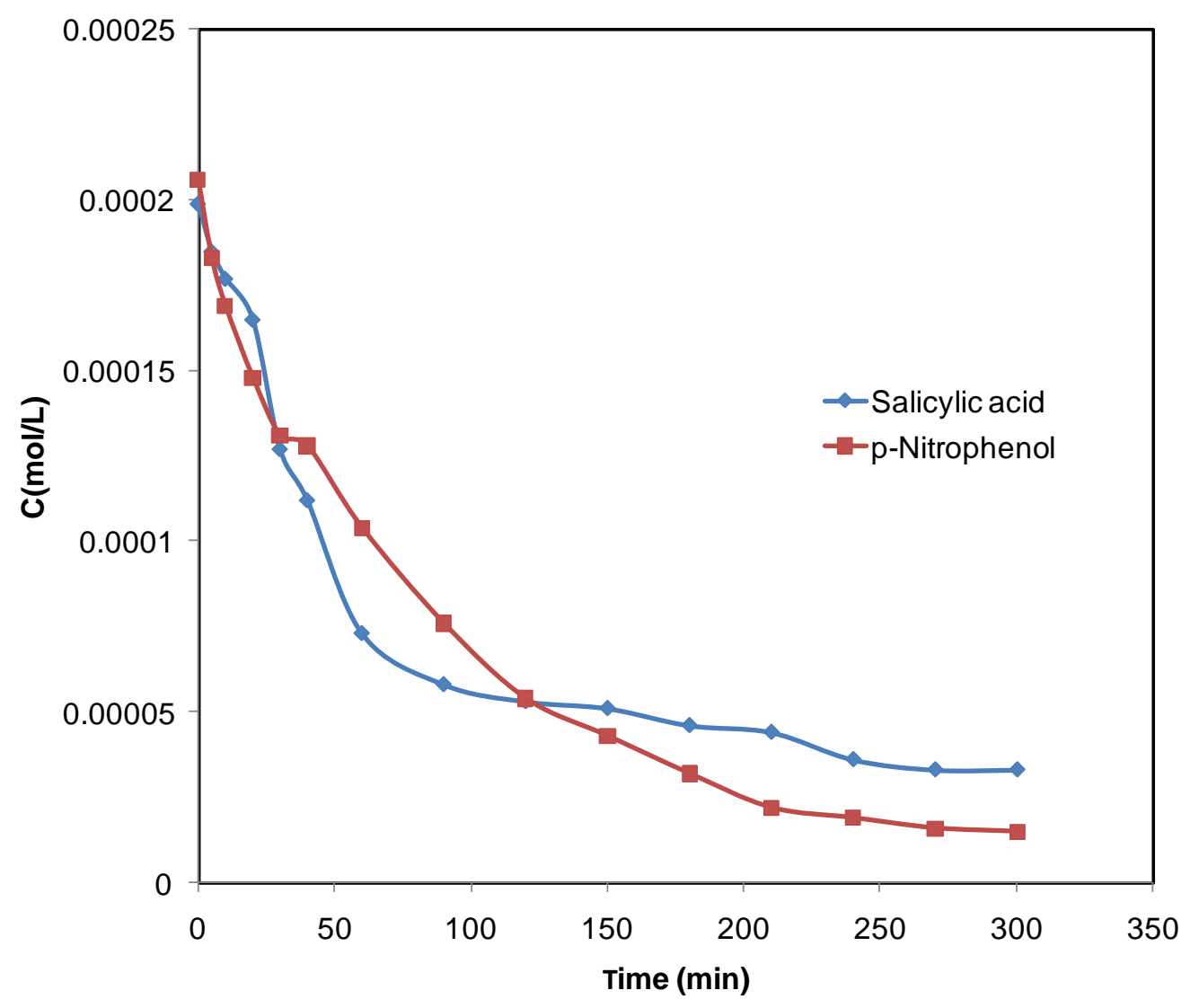

Fig. 6(c)

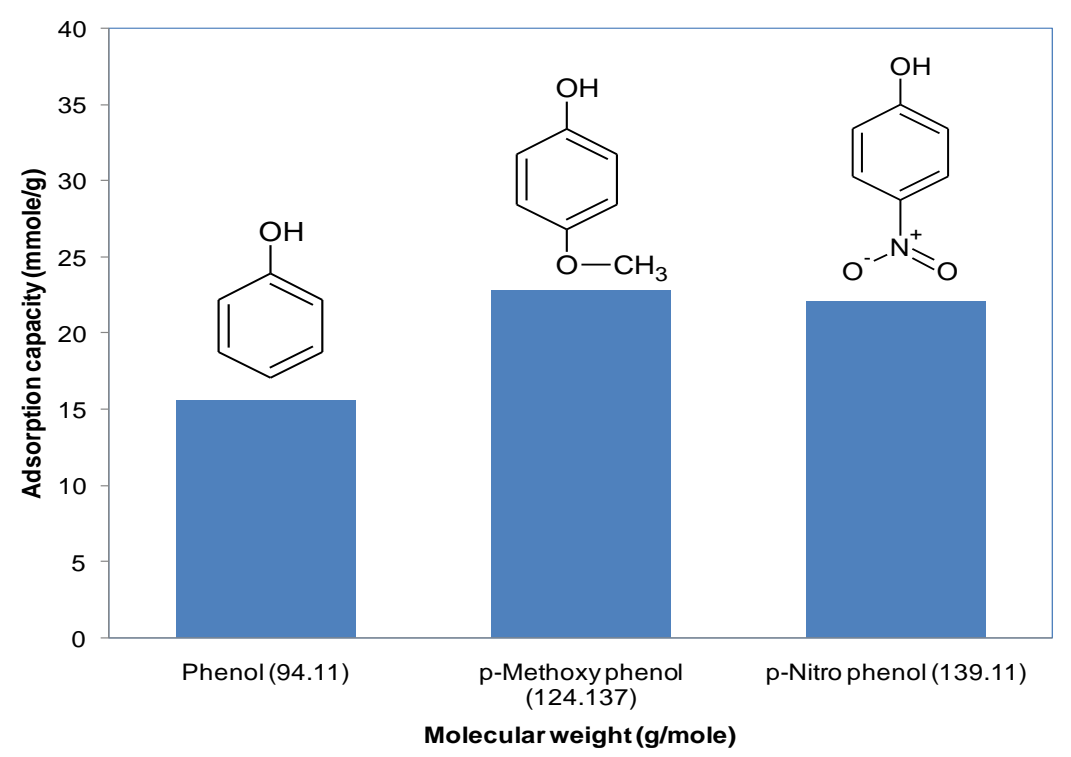

Fig.7. 